\section{Benefits of a diet with walnuts in Alzheimer's disease}

Izheimer's disease is a severe State Institute for Basic Research in neurodegenerative disease that Developmental Disabilities, New York, the course of 5 to 20 ears the course of 5 to 20 years, patients language skills and cognitive function, with great impact on their quality of life. Although Alzheimer's disease affects more than five million Americans, there is currently no known cure.

This condition develops in the brain when toxic amyloid beta-protein accumulates as amyloid plaques, which surround bran cells, leading to cell death. Although the exact mechanisms have not yet beee fully uncovered, there is some evidence to point in the direction of increased oxidative stress induced by amyloid betaprotein as one of the culprits.

The good news is that several studies suggest that consumption of walnu in the diet may significantly slow Dr Abha Chauhan from the New York USA, suggests this is likely due to a high antioxidant* content (including gamma tocopherol (vitamin E), flavonoids and lant [alpha-linolenic acid (ALA) $25 \mathrm{~g} / \mathrm{oz}$ in walnuts. She has spent the past few years tying to determine whether a diet with walnuts can help to reduce the risk of dementia or slow the onset or progression of Alzheimer's disease, and its underlying mechanisms.

\section{WALNUTS PROTECT}

AGAINST ALZHEIMER'S

AMYLOID BETA-PROTEININDUCED OXIDATIVE STRESS AND CELL DEATH The first step on this journey was Atztablish the link between walnuts, For this, the researchd oxidative stess. cultured in a petri dish to which synth cultired in a petri dish to which synthetic
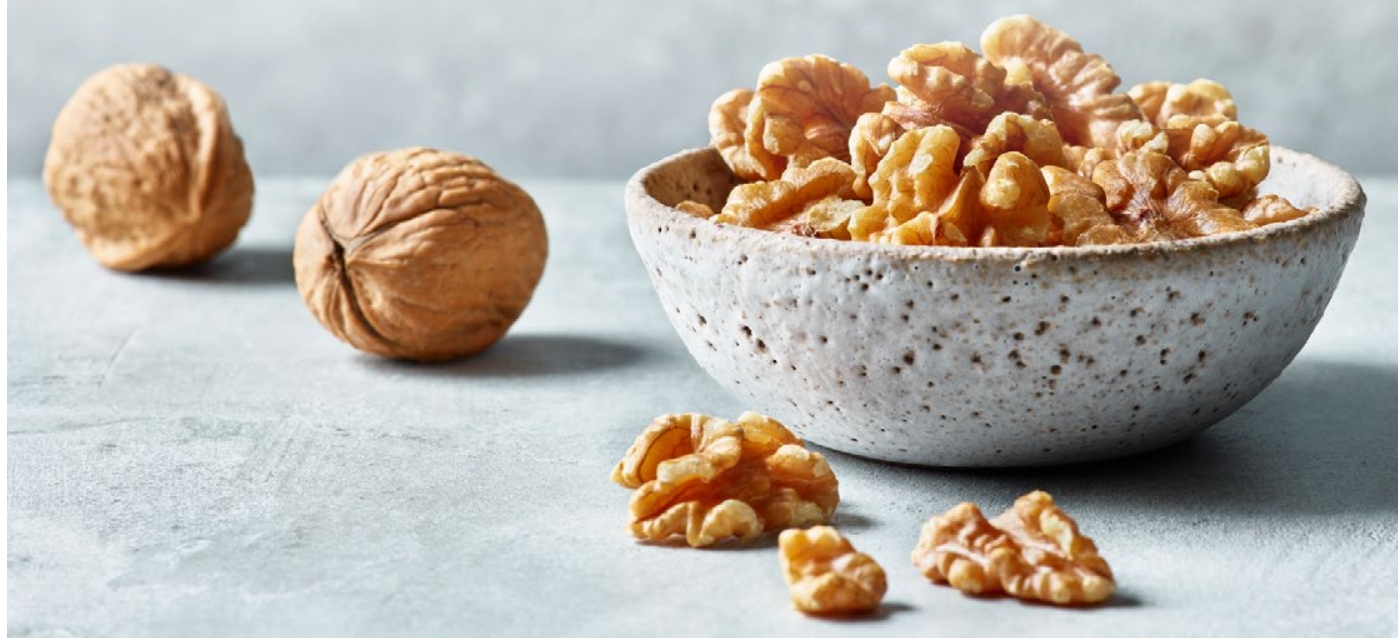

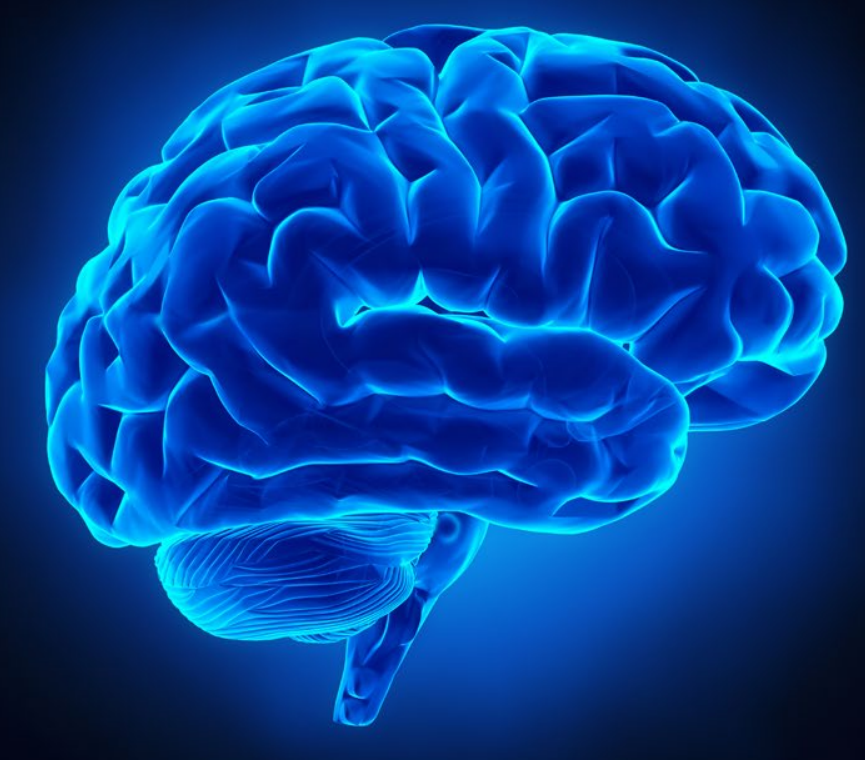

mimic the conditions of Alzheimer's in high levels of lipid peroxidation DNA fragmentation and production of free radicals, eventually leading to cell death. In the cells with added walnut extract, however, the effects of the synthetic amyloid beta-protein were reduced

These results indicated that walnuts have the ability to scavenge free radicals, limit oxidative stress and prevent cell death induced by the amyloid betaof Alzheimer's.

Researchers suggest this is due to a antioxidants present state, says Dr Chauhan. "A diet rich In walnuts may therefore reduce amy beta-protein-mediated cytotoxicity, neuronal loss, and the risk of developing nd cell mortality was lower. Alzheimer's.

\section{Although Alzheimer's disease affects} more than five million Americans, there is currently no known cure.

政 foods with anti-oxidative properties. "This effect of walnut extract could be due to the active compounds present in walnuts, which may increase the capacity of endogenous antioxidant defence and may modulate the cellular redox and control mice were comparable in the contents of protein, carbohydrate Walnut supplementation allowed the Alzheimer's mice to show better learning and motor skills when exploring a maze. In fact, these animals showed less stress and anxiety, made fewer mistakes and escaped faster than their counterparts on a diet without walhut supplementation. DrChauhan explains: "Diets enriched
with walnuts may have provided

improved memory and cognitive function in the AD-tg mice in our study because ingredients to reduce oxidative stress and inflammation."

Based on these results, it's reasonable to suggest that supplementation with walnuts may help in reducing the risk of developing Alzheimer's disease, delaying its onset and/or slowing ts progression due to the antioxidant chects of differen nuts. At the very least, these results indicate that it may be worth studies in humans.

Walnuts offer a variety of antioxidants $(3.721 \mathrm{mmol} / 0 z)$, including polyphenols $(69.3+16.5 \mathrm{\mu mol}$ catechin equivalents/g) and gamma tocopherol $(5.91 \mathrm{mg} / \mathrm{oz})$. 


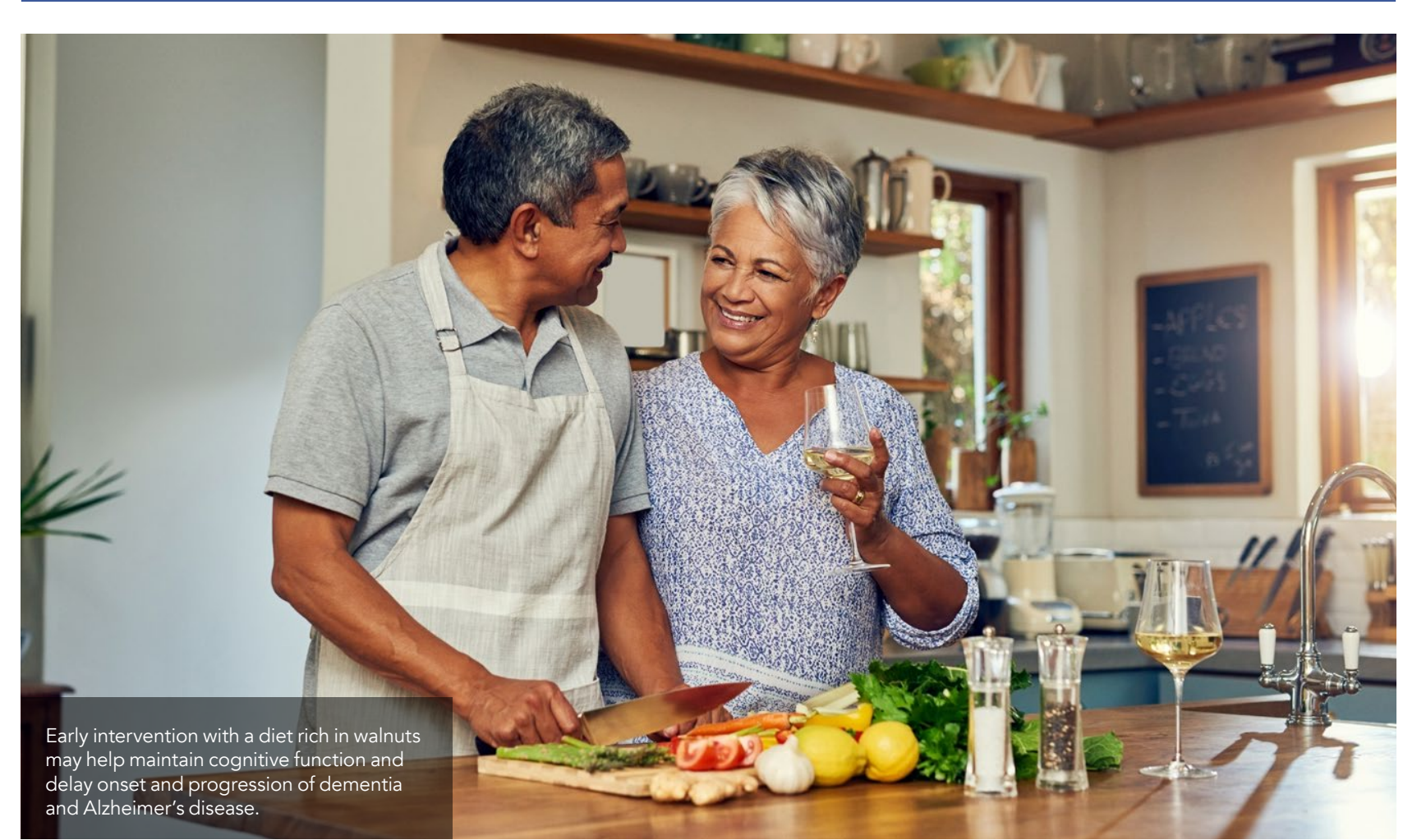

It's difficult to say at this stage what exactly in the walnut is responsible for these benefits, but in addition to fatty acid) may also be a contributing factor. While most nuts contain

serotonin and dopamine concentrations, as well as modulating key inflammatory and immune functions

The authors further explored the mechanism behind walnuts' beneficial

Walnuts in the diet reduce oxidative stress and improve the body's defence mechanisms in Alzheimer's mice.

monounsaturated fats, only walnuts consist primarily of polyunsaturated fat, of which ALA is the main constituent. fatty acids, important for regulating

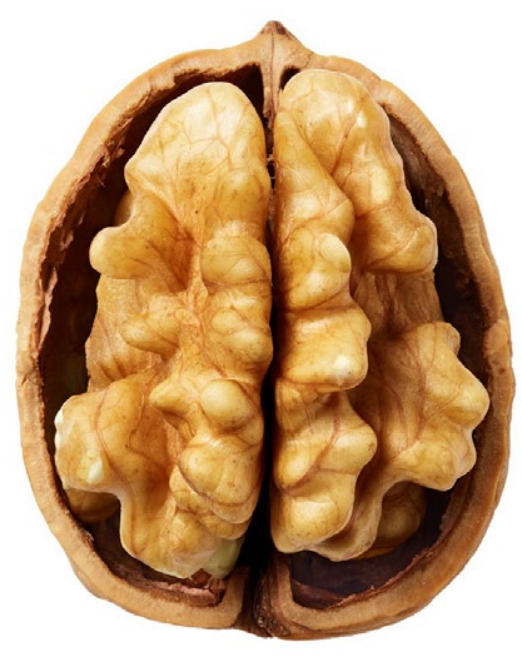
Alzheime's disease Dr Chac of research demonstrates that $6 \%$ or $9 \%$ walnuts in the diet reduce oxidative stress and improve the body's defonce mechanisms in Alzheimer's mice.

As expected, dietary supplementation with walnuts decreased the levels of free radicals, which in turn led to a Free radicals are highly unstable and easily react with lipids and proteins. Lipids, for example, are an ideal target for free radical attack, leading to the production of unstable compounds th cause havoc inside cells.

These results can be explained by the increase detected in a few specia antioxidant enzymes with the ability
to fight free radicals in AD-tg mice on a diet with walnuts. In contrast, mice that did not receive walnut in their diets showed increased free radical production and oxidation of lipids and proteins, which was accompanied by decreased activities of protective

From these results, it seems that walnut supplementation can have a beneficia effect not only by limiting oxidative stress, leading to less damage to lipids and proteins present in cells, but also by boosting the body's ability to fight free radical production. lt's a 2-in-1 win. finding suggest tha supplementation with walnuts in the diet improved endogenous antioxidant function associated with removing accumulated between free radicals and antioxidants, thus reducing oxidative damage to lipids and proteins in AD-tg mice."

It's reasonable to speculate that early intervention with a diet rich in walnuts may help maintain cognitive function and delay onset and progression of dementia and Alzheimer's disease by limiting damage caused by free radicals and enhancing the body's free radicals and improving the balance

\section{A. Behind the Research}

\section{Dr Abha Chauhan}

321. E: abha.chauhan@opwdd.ny.gov

\section{Research Objectives}

DrChauhan's research is focused on oxidative stress, mitochondrial dysfunction, signal transduction, membran

\section{Detail}

Abha Chauhan, Ph.D.

A

NYS Institute for Basic Research in Developmental Disabilities

Staten Island, New York 10314

Bio

Abha Chauhan, Ph.D., is the Head of the Developmental Neuroscience Laboratory at the New York State Institute for Basic Research in Developmental Disabilities (IBR) and adjunct professor of the Neuroscience doctoral program at the City University of New York. She has over

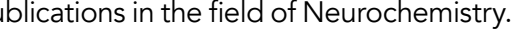

Funding

Temmission, New York State Office for People with Developmental Disabilities

\section{Collaborators}

arch in Developmental

Staten Island, New York

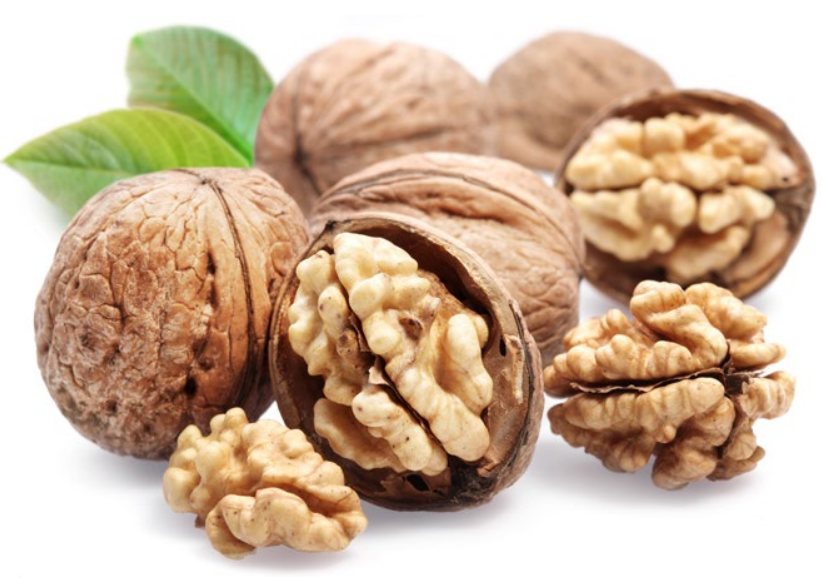

\section{References}

Muthaiyah B, Essa MM, Chauhan V, Chauhan A (2011). Protective effects of walnut extract against amyloid beta peptide-induced cell death and oxidative stress in PC12 cells,
Neurochem Res. 36(11):2096-103. Available at: https://www. Neurochem Res. 36(11):2096-103. Available at: https://www.
ncbi.nlm.nih. gov/pmc/articles/PMC3183245/ [Accessed 18th ncbi.nlm.nih.

Muthaiyah B, Essa M, Lee M, Chauhan V, Kulbir K, Chauhan A (2014) Dietary Supplementation of Walnuts Improves Model of Alzheimer's Disn S A

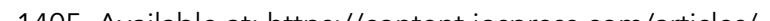
journal-of-alzheimers-diseasejad140675. Accessed 18th April 2019].

Pandareesh MD, Chauhan V, Chauhan A (2018). Walnut Supplementation in the Diet Reduces Oxidative Damage and Improves Antioxidant Status in Transgenic Mouse Model of Alzheimer's Disease. J Alzheimers Dis. 64(4):1295-1305. Available at: https://www.ncbi.nlm.nih.gov/pmc/articles/ PMC6087457/ [Accessed 18th April 2019].

\section{Personal Response}

Your research has demonstrated protective effects of walnuts on cognitive functioning. What is next for your work?

II Accumulation of amyloid beta-protein (AB) is clearance. Amyloid precursor protein (APP) is processed by non-amyloidogenic and amyloidogenic pathways. In non-amyloidogenic pathway, APP is cleaved by a-secretase to yield a soluble N-terminal fragment (sAPPQ), which is APP is cleaved by $\beta$-secretase with subsequent cleavage by $\gamma$-secretase, thus releasing A $A$. Therefore, activation of $\alpha$-secretase and inhibition of $\beta$ - and $\gamma$-secretases can inhibit $A \beta$ production. In addition, the clearance of $A \beta$ is accomplished by its proteolytic degradation. We would production and/or increase its degradation by props thus reducing the levels of brain $A \beta$ in Alzheimer's transgenic mice. 